\title{
Strand 6B deformation and residues exposure towards N-terminal end of helix $B$ during proteinase inhibition by Serpins
}

\author{
Poonam Singh \& Mohamad Aman Jairajpuri*
}

Department of Biosciences, Jamia Millia Islamia University, Jamia Nagar, New Delhi-110025, India; Mohamad Aman Jairajpuri- Phone: 0919971259812; Fax: 091-11-26980229; Email: m_jairajpuri.bi@jmi.ac.in; *Corresponding author

\begin{abstract}
:
Serine Protease inhibitors (Serpins) like antithrombin, antitrypsin, neuroserpin, antichymotrypsin, protein C-inhibitor and plasminogen activator inhibitor is involved in important biological functions like blood coagulation, fibrinolysis, inflammation, cell migration and complement activation. Serpins native state is metastable, which undergoes transformation to a more stable state during the process of protease inhibition. Serpins are prone to conformation defects, however little is known about the factors and mechanisms which promote its conformational change and misfolding. Helix B region in serpins is with several point mutations which result in pathological conditions due to polymerization. Helix B analysis for residue burial and cavity was undertaken to understand its role in serpin structure function. A structural overlap and an accessible surface area analysis showed the deformation of strand 6B and exposure of helix B at $\mathrm{N}$-terminal end in cleaved conformation but not in the native and latent conformation of various inhibitory serpins. A cleaved polymer like conformation of antitrypsin also showed deformation of s6B and helix B exposure. Cavity analysis showed that helix B residues were part of the largest cavity in most of the serpins in the native state which increase in size during the transformation to cleaved and latent states. These data for the first time show the importance of strand 6B deformation and exposure of helix B in smooth insertion of the reactive center loop during serpin inhibition and indicate that helix B exposure due to variants may increase its polymer propensity.
\end{abstract}

Keywords: serine protease inhibitor; protein aggregation and folding; reactive center loop; $\alpha 1$-antitrypsin; shutter domain; accessible surface area; CASTp.

Abbreviations: serpin, serine protease inhibitors; RCL, reactive center loop; ASA, accessible surface area;

\section{Background:}

Serine protease inhibitors (Serpins) are a unique superfamily of protease inhibitor, which is involved in important biological processes like blood coagulation (antithrombin and heparin cofactor II), fibrinolysis (protein C inhibitor), inflammation ( $\alpha 1$-antitrypsin and antichymotrypsin), cell migration (plasminogen activator inhibitor) and complement activation (C1-inhibitor) [1-2]. Serpins have common secondary fold, which is defined by at least $30 \%$ sequence identity and constitutes seven $\alpha$-helices and three $\beta$ sheets. Serpin in the native form is in the metastable conformation which undergoes a transition to a more stable structure during the process of protease inhibition [3]. During the inhibition mechanism, the RCL (Reactive Center Loop) bound protease is translocated to more than $70 \mathrm{~A}^{\circ}$ away on the opposite site [4]. Large scale conformation change involved in serpin inhibition mechanism also increases its tendency to polymerize. Pathological molecular linkages are produced when variants allow deformation of the A-sheet conformation which aberrantly opens the A-sheet [5]. Based on the incorporation of part of RCL peptide between strand $3 \mathrm{~A}$ and strand $5 \mathrm{~A}$ and its capability to block polymerization, it was proposed that polymerization in serpins occurs due to the insertion of RCL of one molecule into the beta-sheet A of another to form a loop-sheet linkage [6-7]

Shutter region constituting F-helix, B-helix, strands s3A and s5A of $\beta$ sheet A play an important role in stability and function in serpins [1, 7-8].
Helix B interacts with the $\beta$-sheet $\mathrm{A}$ amino acids which are conserved among various serpins. Helix B is located at the upper portion of the shutter region where RCL inserts as s4A. Helix B mutations in $\alpha 1$ antichymotrypsin (Leu55Pro) and $\alpha 1$-antitrypsin (Phe51Leu, Ser53Phe and Val55Pro) can cause lung (emphysema) and liver diseases (cirrhosis). Protein C-inhibitor (Ser52Phe and Ser54Leu) and antithrombin (Pro80Ser/Thr, Thr85Met/Lys, Cys95Arg and Leu99Phe) have mutations which can result in angioedema and thrombosis, similarly in neuroserpin Ser53Pro, Ser49Pro, Ser56Arg and Ser52Arg are linked to hereditary disorder called familial encephalopathy with neuroserpin inclusion bodies (FENIB) [9-12]. It has been shown that network of residues dynamically coupled with each other, especially inside cavities and local and global stability of these regions mediate inhibitory activity [13]. Increase polymerization in the helix $\mathrm{B}$ region might be due its presence in cavity that is involved in RCL translocation during the protease inhibition mechanism.

In this study, we for the first time hypothesize that strand $6 \mathrm{~B}$ deformation linked helix $\mathrm{B}$ exposure at $\mathrm{N}$-terminal end and cavity alteration help in smooth insertion of the RCL in inhibitory serpin. Natural variants of serpins might distort stand $6 \mathrm{~B}$ or increase helix $\mathrm{B}$ flexibility to make it prone to polymerization. 


\section{Bioinformation}

\section{Methodology:}

Structural Analysis:

We selected representative inhibitory serpins with available native, cleaved and latent PDB structures for structural overlap analysis. We used Chimera for superimposing the structures of $\alpha 1$-antitrypsin, antichymotripsin, protein $\mathrm{C}$ inhibitor and neuroserpin. Match Maker extension of Chimera which constructs pair-wise sequence alignments was used for superimposing the serpin structures [14]. The default settings identifies the best matching chains based on alignment scores, we have used NeedlemanWunsch algorithm with BLOSUM-62 and 30\% weighting of the secondary structure term.

PDB structures used for structural overalaps are as follows: antithrombin [2.6̊̊ native: pdb.1E05(I) and $3.2 \AA$ cleaved: pdb.1ATH(A) from human

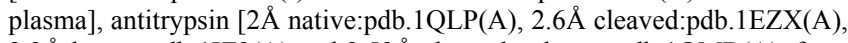
$2.2 \AA$ latent pdb.1IZ2(A) and $2.59 \AA$ cleaved polymer:pdb.1QMB(A), from human plasma], neuroserpin [2.08 $\AA$ native:pdb.3FGQ(A) and $1.79 \AA$ cleaved:pdb.3FO2(A) from human], antichymotrypsin [2.26̊ native like delta conformation: pdb.1QMN(A) and $2.9 \AA$ cleaved:pdb.4CAA(A), from human], and protein $\mathrm{C}$ Inhibitor [2.30 $\AA$ native: pdb.2HI9(A) and $2.40 \AA$ cleaved:pdb.1LQ8(A) from human plasma]

\section{Computation of Accessible Surface Area:}

We carried out solvent accessibility analysis on native and cleaved states of antitrypsin, antithrombin, neurosrpin, antichymotrypsin and protein $C$ inhibitor by using DSSP (Dictionary of Secondary Structure of Proteins) tool. Accessible surface area (ASA) is important for elucidating atomic surface area of a molecule (protein, DNA, etc.) that is accessible to a solvent, and is usually expressed in $\AA^{2}$ (square Angstroms). ASA is calculated using the 'rolling ball' algorithm which uses a sphere (representing the solvent) of a particular radius to 'probe' the surface of the molecule. A typical value of a 'probe radius' is $1.4 \AA$, which approximates the radius of a water molecule $[15,16]$. Extended states of Ala-X-Ala for every residue $\mathrm{X}$ has been used (assuming that the absolute values include side chain and backbone surface area) [17]. The corresponding values are (in $\AA^{2}$ ) 110.2 (Ala), 144.1 (Asp), 140.4 (Cys), 174.7 (Glu), 200.7 (Phe), 78.7 (Gly), 181.9 (His), 185.0 (Ile), 205.7 (Lys), 183.1 (Leu), 200.1 (Met),146.4 (Asn), 141.9 (Pro), 178.6 (Gln), 229.0 (Arg), 117.2 (Ser), 138.7 (Thr), 153.7 (Val), 240.5 (Trp), and 213.7 (Tyr) respectively.

\section{Cavity analysis using CASTp:}

CASTp was used to study surface features, functional regions and roles of important residues of different serpin conformations like native, latent, cleaved and polymeric state. CASTp maps surface pockets and interior voids or other regions of the PDB structures. It also gives an interactive visualization of computed pockets [18]. CASTp uses the weighted Delaunay triangulation and the alpha complex for shape measurements. It provides identification and measurements of surface accessible pockets as well as interior inaccessible cavities, for proteins and other molecules. It measures analytically the area and volume of each pocket and cavity, both in solvent accessible surface (SA, Richards' surface) and molecular surface (MS, Connolly's surface) [19]. All hetero atoms treated as ligands are automatically removed from calculation, which includes solvent water molecules.

\section{Calculation of free energy change upon point mutations:}

I mutant 2.0 analysis was carried out at temp $25^{\circ} \mathrm{C}$ and $\mathrm{pH} 7.0$. Free energy changes upon single point mutations were computed on different structures of our study. It is a support vector machine (SVM)-based tool for the automatic prediction of protein stability changes upon single point mutations [20].

\section{Results and Discussion:}

Structural comparison of helix B in inhibitory serpins:

Cleaved state of a serpin represents the inhibitory conformation where after cleavage reactive center loop inserts between strand $3 \mathrm{~A}$ and strand $5 \mathrm{~A}$ as strand $4 \mathrm{~A}$ of $\beta$-sheet $\mathrm{A}$. Structural overlap of strand $6 \mathrm{~B}$ and helix B in the cleaved and native state of inhibitory serpins like antitrypsin, antichymotrypsin, neuroserpin and protein $\mathrm{C}$ inhibitor is shown in Figure 1. The comparison showed strand $6 \mathrm{~B}$ was deformed in the cleaved state but not in the native state. In antitrypsin we see loss of one turn at the Cterminal end of the helix B in the cleaved structure (Figure 1) along with the deformation of the strand $6 \mathrm{~B}$. Antithrombin is a heparin binding anticoagulant inhibitor of factor $\mathrm{Xa}$ and thrombin that showed no deformation of strand $6 \mathrm{~B}$ in the cleaved state (data not shown). Latent conformation of serpins is a loop inserted conformation without RCL cleavage, no strand $6 \mathrm{~B}$ deformation was found in this state (data not shown).

\section{Accessible surface area analysis of helix $\mathrm{B}$ :}

Helix B is wrapped around the strand $2 \mathrm{~A}, 3 \mathrm{~A}$ and $5 \mathrm{~A}$ of the $\beta$ sheet $\mathrm{A}$ with many conserved residues involved in hydrogen bonding interactions. We hypothesize that for the smooth insertion of the RCL helix B has to be exposed, and strand 6B deformation may aid in helix $\mathrm{B}$ backbone flexibility during the RCL translocation. To analyze helix B exposure in the cleaved and latent state we did residue burial analysis by using Accessible Surface Area (ASA) analysis. Burial of residue relative to the same residue in Ala-X-Ala tripeptide can be estimated and a completely buried residue versus an exposed residue can be distinguished by values of $5 \%$ to $25 \%$ (or more) [20]. A comparison of the residue burial in the native and the cleaved structures of serpins like antitrypsin, neuroserpin, antichymotrypsin and protein $\mathrm{C}$ inhibitor is shown in Figure 2. ASA analysis clearly indicated that conformational deformation of strand $6 \mathrm{~B}$ has resulted in the exposure of residues in the $\mathrm{N}$-terminal end of the helix $\mathrm{B}$ in most of the cleaved serpins but not in the native and latent conformations (data not shown). There was a remarkable similarity in the exposure pattern of the cleaved state among various serpins, Asn49 in antitrypsin and antichymotrypsin and their homologue (Asn45 in neuroserpin and protein $\mathrm{C}$ inhibitor) were all fully exposed in the cleaved conformation. Helix B exposure was until Val55 in antitrypsin and their homologues like Val51 in Protein C inhibitor, Leu51 in neuroserpin and Leu55 in antichymotrypsin (Figure 2). In antitrypsin Asn45 near strand $6 \mathrm{~B}$ is highly hydrogen bonded with the edge of C-terminal residues like Val389, Asn390 and Gln393. Large changes in burial pattern in a conserved Asn in each serpins (Figure 2) may lead to the deformation of the strand $6 \mathrm{~B}$ and moving away of helix B from sheet A. Antithrombin binds heparin cofactor for maximum inhibition of serpine proteases, no s6B deformation or helix B exposure was observed in this serpin probably because of differences in mechanism arising due to cofactor based activation. Latent state is a loop inserted conformation where stand $6 \mathrm{~B}$ deformation and helix $\mathrm{B}$ exposure is not seen indicating that these structural changes are unique to RCL insertion during protease inhibition mechanism.

\section{Helix B is part of the largest cavity in most serpins:}

Surface cavity contributes to metastability of antitrypsin and cavities near the $\beta$-sheet $\mathrm{A}$ have been shown to be important in regulating the inhibitory activity [21, 22]. We did cavity analysis of various serpins to find out if the helix B residues are indeed part of cavity and to assess their volume and area. The results of cavity analysis are summarized in Table 1. Analysis of the native state of antitrypsin (pdb.1QLP) showed that there is a total of 66 cavities where the volume of the largest cavity was $526 \AA^{3}$ and it had an area of about $459 \AA^{2}$. This cavity predominantly included residues, which are part of helix B (Ser53, Val55, Ser56 T59 and Ala60), along with residues at $\mathrm{N}$-terminal and $\mathrm{C}$ terminal end of helix $\mathrm{D}$ and Asn186 and Ile188 from s3A. In the latent structure (pdb.1IZ2) of antitrypsin the biggest cavity had a volume $\left(701 \AA^{3}\right)$ which is slightly greater than the native. As shown in Table 1 (see Supplementary material) helix B is part of the biggest cavity in most of the native state of the serpins analyzed in our study. Remarkably, our analysis showed that in going from native to cleaved (pdb.7API) and polymeric (pdb.1D5S) states massive transition takes place in the cavity size where the size of the largest cavity increased from $526 \AA^{3}$ in native to a volume of $4761 \AA^{3}$ in the polymer state and $5339 \AA^{3}$ in the cleaved state. Large volume of the cavities in cleaved and polymeric conformations might be exaggerated 
because both structures have the loop of another molecule inserted between strand $3 \mathrm{~A}$ and $5 \mathrm{~A}$, and the analysis was performed on monomer. Large cavity formation in the upper portion of the shutter region in cleaved and polymeric conformations but not in native could have implications in targeting this area for hindering polymerization.

Importance of helix B hydrogen bond interaction and serpin stability: A comparison of cleaved and native conformation of antitrypsin showed that the $\mathrm{B}$-sheet $\mathrm{A}$ in the central portion of the $\mathrm{s} 3 \mathrm{~A}$ and $\mathrm{s} 5 \mathrm{~A}$ and helix $\mathrm{B}$ is linked by a network of hydrogen bond, which blocks the loop insertion to the lower part of the B-sheet A (data not shown). In the native state of antitrypsin Tyr297 from strand 6A forms a hydrogen bond with the backbone nitrogen of Phe51 from helix B, His334 from strand 5A hydrogen bonds with Ser53 backbone nitrogen and Asn186 in strand $3 \mathrm{~A}$ is hydrogen bonded to Ser56 in helix B. During RCL insertion s2A and s3A shifts in the opposite direction and both His334 and Tyr297 rotate in cleaved conformation leading to more exposure of helix B. Phe51 is lifted upwards in both the states along with the formation of hydrophobic contact between His334 and Phe52. Helix B is wrapped over strand A and for the $\mathrm{RCL}$ to move in as strand $4 \mathrm{~A}$ the helix $\mathrm{B}$ is loosened due to strand $6 \mathrm{~B}$ deformation. This loosening of helix $\mathrm{B}$ allows the reactive center loop to insert smoothly.

Mutations causing alteration in the hydrogen bonding network will cause destabilization allowing helix $\mathrm{B}$ to be exposed without cleavage resulting in insertion of RCL of another molecule into the $\beta$-sheet $A$. As shown in Figure 2 helix B amino acids are deeply buried in native state in most of the serpins which are exposed in the cleaved state. Polymerization variant of serpins due to their deep burial and presence inside the cavity can shift the overall stability of the protein which might explain its increased polymer propensity. We did an analysis of free energy change upon point mutation using I mutant 2.0 program at temp $25^{\circ} \mathrm{C}$ and $\mathrm{pH} 7.0$ and the results indicate that most of the variants in the helix $\mathrm{B}$ region were destabilizing with $\Delta \Delta \mathrm{G}$ values ranging from $-0.4 \mathrm{Kcal} / \mathrm{mol}$ to -3.0 $\mathrm{Kcal} / \mathrm{mol}$ (data not shown). The results clearly indicate that the deeply burial of helix B residues and its presence inside cavities can cause the global destabilization in the variants of serpins which may result in increased conformational flexibility.

\section{Conclusion:}

In conclusion, our data for the first time shows a plausible role of helix B in the mechanism of serpin inhibition and polymer formation across many inhibitory serpins. The results indicate that deformation of the strand $6 \mathrm{~B}$ at the edge of helix B might confer extra flexibility to the N-terminal end of helix $\mathrm{B}$ residue to lift the helix so that strand $4 \mathrm{~A}$ can smoothly pass further down during the process of protease inhibition.

Proposed sequence of events during serpin inhibition mechanism is hypothesized to be as follows: 1) Binding of protease results in partial insertion of RCL at the top of $\beta$-sheet A, 2) which leads to the loss of hydrogen bonding interactions between a conserved Asn near strand $6 \mathrm{~B}$ and $\mathrm{C}$-terminal residues, which results in deformation of stand 6B. 3) This deformation helps in helix B exposure, 4) which facilitate further insertion of the RCL as $\mathrm{s} 4 \mathrm{~A}$ and proteinase translocation to the opposite end of the $\beta$ sheet A.

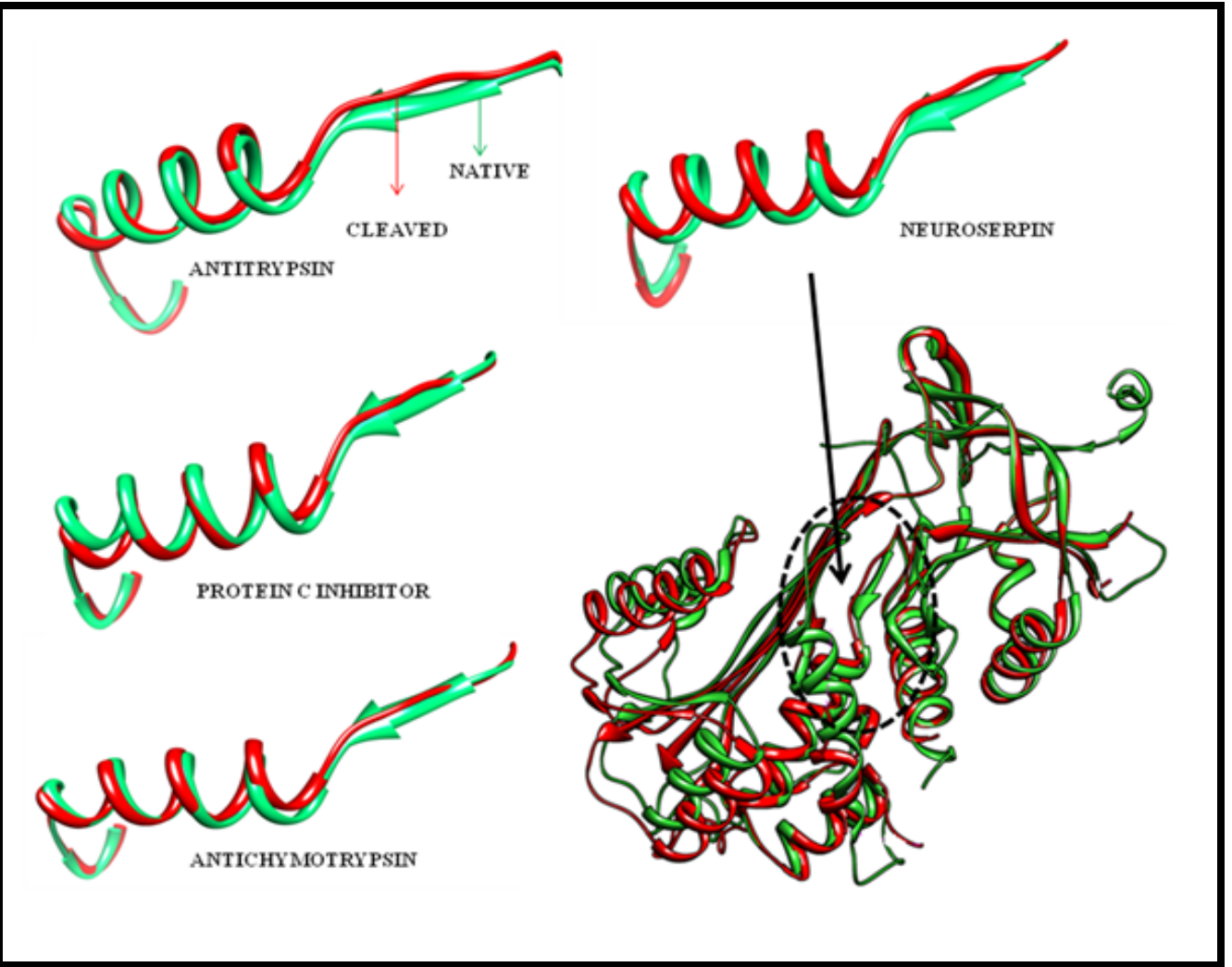

Figure 1: Structural overlap of the native and cleaved conformation of strand $6 \mathrm{~B}$ and helix $\mathrm{B}$ in serpins: Illustrations show the overlap of cleaved and native conformations in $\alpha 1$-antitrypsin, neuroserpin, protein $\mathrm{C}$ inhibitor and antichymotrypsin indicating the deformation of strand $6 \mathrm{~B}$. The analysis was

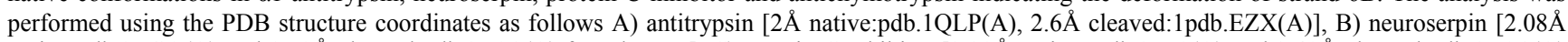

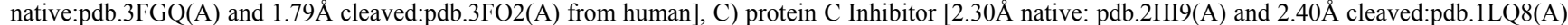

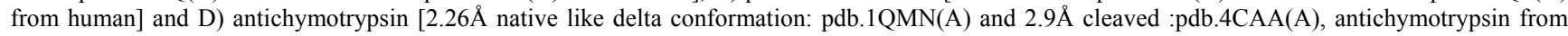
human]. Chimera software was used for creating the comparison and visualization. 


\section{Bioinformation}

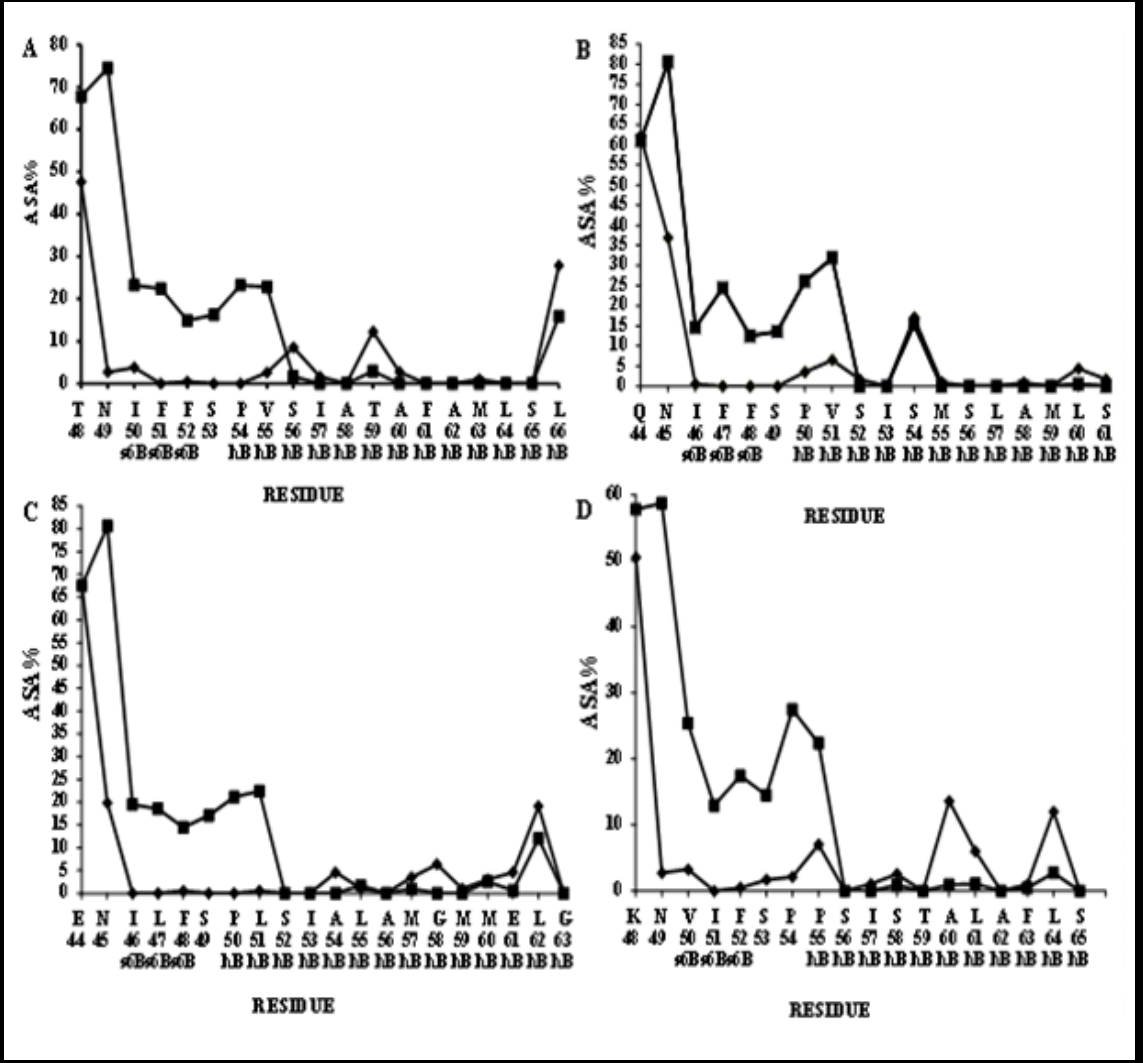

Figure 2: Strand 6B and helix B burial analysis in serpins: A comparison of the burial in stand 6B and helix B region in various cleaved (- - -) and native $(-\downarrow)$ conformations of inhibitory serpins. ASA values were determined from DSSP algorithm as detailed in Methodology section. The figures and their PDB codes used for each graph are detailed in methodology section and in Figure 1.

\section{Acknowledgements:}

This research was supported by grants from Department of Biotechnology and University Grant Commission, Government of India. PS is supported by a grant from UGC. We acknowledge Protein Data Bank, Brookhaven National Lab, for protein structure coordinates.

\section{References:}

[1] GL Devlin \& SP Bottomley, Frontier in Bioscience 10: 288 (2005) [PMID: 15574369]

[2] PE Stein \& RW Carrell, Structural Biology 2: 96 (1995) [PMID: 7749926]

[3] JA Huntington et al. Nature 407: 923 (2000) [PMID: 11057674]

[4] AE Mast et al. Biochemistry 30: 1723 (1991) [PMID: 1704258]

[5] DA Lomas et al. Biochem Soc Trans 33: 321 (2005) [PMID: 15787598]

[6] E Stratikos \& PG Gettins, Proc Natl Acad Sci USA. 94: 453 (1997) [PMID: 9012804]

[7] MA Dunstone et al. Protein Science 9: 417 (2000) [PMID: 10716194]

[8] B Gooptu \& DA Lomas, Ann. Rev. of Biochem 78: 147 (2009) [PMID: 19245336]
[9] DA Lomas \& RW Carrell, Nat. Rev Genet 73: 59 (2002) [PMID: 12360234]

[10] DC Crowther, Hum. Mutat Review 20: 1 (2002) [PMID: 12112652]

[11] JC Whisstock et al. J Mol Biol. 295: 651 (2000) [PMID: 10623554]

[12] RW Carrell, Trends in Cell Biology 15: 574 (2005) [PMID: 16202603]

[13] T Sengupta et al. Biochemistry 48: 8233 (2009) [PMID: 19624115]

[14] EF Pettersen et al. J. Comput. Chem. 13: 1605 (2004) [PMID: $15264254]$

[15] J Mihel et al. BMC Struct Biol. 8: 21 (2008) [PMID: 18400099]

[16] S Ahmad, et al. BMC Bioinformatics 5: 51 (2004) [PMID: 15119964]

[17] SJ Hubbard \& JM Thornton, J Mol Biol 220: 507 (1993) [PMID: $1856871]$

[18] TA Binkowski et al. Nucleic Acids Res. 31: 3352 (2003) [PMID: 12824325]

[19] J Liang et al. Proteins 33: 1 (1998) [PMID: 9741840]

[20] MM Gromiha et al. Protein Eng 7: 549 (1999) [PMID: 10436080]

[21] PR Elliot et al. Protein Sciences 9: 1274 (2000) [PMID :10933492]

[22] $\mathrm{H}$ Im et al. The Journal of Biological Chemistry 274: 11072 (1999) [PMID :10196190]

Edited by K Gunasekaran

Citation: Singh \& Jairajpuri, Bioinformation 5(8): 315-319 (2010) License statement: This is an open-access article, which permits unrestricted use, distribution, and reproduction in any medium, for noncommercial purposes, provided the original author and source are credited. 


\section{Supplementary material:}

Table 1: CASTp analysis of Connolly surface area and volume of the largest cavities in different conformational states of antitrypsin: Figure shows the volume and area of the biggest cavities in different conformational states of $\alpha-1$ antitrypsin (pdb codes and structure description has been given above). The cavity identification numbers are arbitrary; they are numbered according to the largest to the smallest consecutively as identified by the CASTp program.

\begin{tabular}{|c|c|c|c|c|}
\hline $\begin{array}{l}\text { Serpin (Pdb code) } \\
\text { (helix B region) }\end{array}$ & $\begin{array}{l}\text { Cavity } \\
\text { ID }\end{array}$ & $\begin{array}{l}\text { MS Area } \\
(\AA 2)\end{array}$ & $\begin{array}{l}\text { MS Volume } \\
(\AA 3)\end{array}$ & $\begin{array}{l}\text { Helix B residues involved } \\
\text { in the largest cavity formation }\end{array}$ \\
\hline Antithrombin (1T1F) (80-90) & 82 & 153 & 183 & T85, M89 (Part of $3^{\text {rd }}$ largest cavity) \\
\hline Antitrypsin (1QLP) (54-66) & 66 & 459 & 526 & $\begin{array}{l}\text { S53, V55, S56, T59, A60, M63 (Part of } \\
\text { largest cavity) }\end{array}$ \\
\hline Neuroserpin (3FGQ) (50-63) & 57 & 615 & 648 & $\begin{array}{l}\text { L51, A54, M57, G58, E61 (Part of largest } \\
\text { cavity) }\end{array}$ \\
\hline $\begin{array}{l}\text { Antichymotrypsin (1YXA) } \\
(75-88)\end{array}$ & 63 & 363 & 278 & S77, A80, L84 (Part of largest cavity) \\
\hline $\begin{array}{l}\text { Heparin Cofactor II (1JMJ) } \\
(138-148)\end{array}$ & $\begin{array}{l}60 \\
58 \\
57\end{array}$ & $\begin{array}{l}164 \\
155 \\
74\end{array}$ & $\begin{array}{l}117 \\
168 \\
54\end{array}$ & $\begin{array}{l}\left.\text { S140 ( } 6^{\text {th }} \text { largest cavity }\right) \\
\text { T141 }\left(8^{\text {th }}\right) \\
\text { A142, M145 }\left(9^{\text {th })}\right.\end{array}$ \\
\hline $\begin{array}{l}\text { Protein C Inhibitor (2HI9) } \\
(50-61)\end{array}$ & 67 & 697 & 1603 & P50, V51, S54 (Part of largest cavity) \\
\hline $\begin{array}{l}\text { Plasminogen Activator } \\
\text { Inhibitor (1DB2) (36-48) }\end{array}$ & 69 & 415 & 514 & $\begin{array}{l}\text { Y37, S41, A44, M45 } \\
\text { (Part of largest cavity) }\end{array}$ \\
\hline \multicolumn{5}{|l|}{ AntitrypsinConformation States } \\
\hline Native (1QLP) & 66 & 459 & 526 & S53, V55, S56, T59, A60, M63 \\
\hline Cleaved (1EZX) & 44 & 2110 & 4830 & T48, N49, I50, F51, S53, F52, P54, V55 \\
\hline Latent (1IZ2) & 58 & 460 & 701 & None \\
\hline Cleaved Polymer (1QMB) & 31 & 1935 & 4228 & T48, N49, I50, F51,F52, S53, P54, V55 \\
\hline
\end{tabular}

${ }^{a}$ Amino acids from helix B which forms a part of particular cavity were identified by the CASTp program.

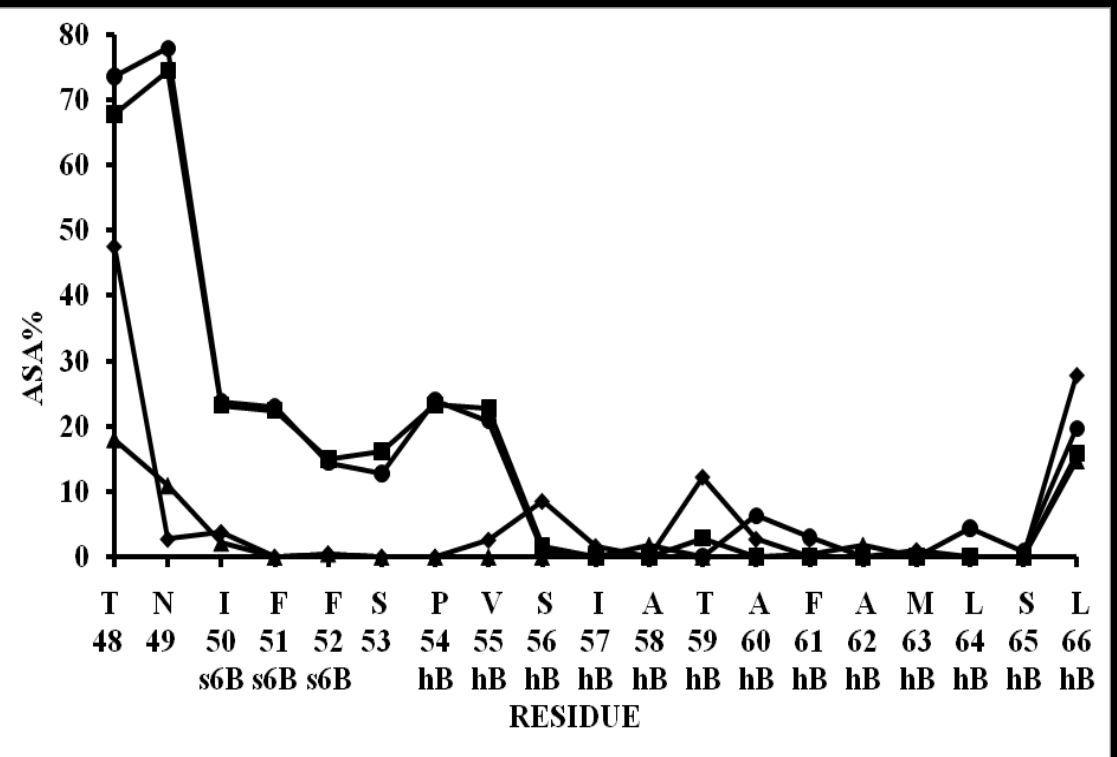

Supplementary Figure 1: Strand 6B and helix B burial analysis in various conformational states of $\alpha 1$-antitrypsin: ASA analyses of strand 6B and helix $\mathrm{B}$ residue was done as detailed in Methodology section. The analysis showed the results with the following conformations of $\alpha 1$-antitrypsin native (- $\mathbf{\Delta}$-),

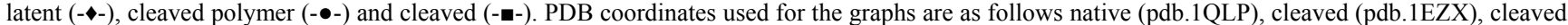
polymer (pdb.1QMB) and latent (1IZ2) structures of the $\alpha 1$-antitrypsin. 